\title{
THE PROPAGATION OF THE TEMPERATURE WAVE IN MYOCARDIUM
}

\author{
Vladyslav Shlykov \\ Department of Biomedical Engineering \\ National Technical University of Ukraine "Igor Sikorsky Kyiv Polytechnic Institute" \\ 37 Peremogy ave., Kyiv, Ukraine, 03056 \\ v.shlykov@kpi.ua
}

\begin{abstract}
The method for estimating the rate of propagation of temperature waves in the myocardium during hypothermia and hyperthermia of the heart based on noninvasive temperature control on the heart surface in conditions of cardiopulmonary bypass is proposed. The solution of the heat conduction equation for temperature waves in the myocardium is presented, which allows determining at each point of the medium the temperature change function, as well as the amplitude and phasing velocity of the temperature wave upon cooling and warming of the heart in conditions of cardiopulmonary bypass. The velocity distribution for the temperature field on the surface of the myocardium and in the depth of tissues during the hypothermia and hyperthermia of the heart is obtained.

The thermographic images of the heart during controlled hypothermia and hyperthermia of the heart under cardiopulmonary bypass are obtained. The study of the conditions for spreading the temperature wave in the myocardium is performed, which allows to improve the means of intraoperative protection of vital organs and tools for controlling its effectiveness. The results of introduction of methods of non-invasive temperature control in cardiac surgery and the process of intraoperative cardiac protection in the temperature range from $+10{ }^{\circ} \mathrm{C}$ to $+38^{\circ} \mathrm{C}$ are presented.
\end{abstract}

Keywords: heart temperature, temperature waves, hypothermia, hyperthermia, cardiopulmonary bypass.

\section{Introduction}

The propagation of temperature waves in the heart during the process of hypothermia and hyperthermia is a periodic change in the temperature distribution in a homogeneous medium - the myocardium, which is associated with periodic fluctuations in the density of the heat flows entering the environment from the contour of cardiopulmonary bypass.

The temperature waves contain information on the properties of the myocardium (heat, temperature, conductivity, density) and the nature of the processes and phenomena that give rise to them (blood flow in the contour of artificial blood circulation, oscillatory processes of the temperature in the operating field, the frequency of rotation of the rollers of the cardiopulmonary bypass). Thermal conductivity is the main mechanism of transferring heat to tissues that are not directly exposed to heat, and the temperature is the main physical value that characterizes all thermal interactions in the myocardium with hypothermia and hyperthermia under artificial blood circulation.

The temperature waves in the myocardium are characterized by some features that distinguish them from electromagnetic and acoustic waves in biological objects. When distributed in biological tissues, the temperature waves are strongly suppressed with penetration, they are characterized by a significant variance - the dependence of the rate of propagation on the frequency of heat sources.

The change in the depth of penetration of the temperature wave depends on the frequency of thermal defectoscopy depending on the frequency, which allows them to be used to detect microcavities in the tissues, disturbance of blood supply and zones of ischemia in the myocardium.

\section{Analysis of literary data and problem statement}

In the conditions of artificial blood flow effects of uneven distribution of temperature in tissues of the human body appear during controlled cooling and warming of the heart and brain.

Useful and important empirical approaches are presented in several well-developed published studies [1-3]. The patterns of heartwarming are based on the method of contrast X-ray examination of blood vessels and a detailed experimental display of isolated hearts. In the above studies, the heat transfer in the myocardium is also measured by various experimental methods. 
In a series of studies to obtain images that show the anisotropy of the tissues of the heart [4-6], the technique of diffusion tensor image was used. Such method of measuring the heat transfer can provide high-quality data that can be used in computer models.

Methods of study of temperature distribution [7], which are based on the empirical approach, can significantly help to verify the parameters of the models developed by the theoretical approach. But this requires correct averaging of the data obtained by displaying isolated samples of the heart. The most important issue of obtaining qualitative information about the coronary flow was not so easy to solve, since open heart operations for controlling the temperature available the only visible side of the heart. At the same time, modern devices and information technologies provide new solutions that can be useful in studies of temperature distribution and distribution of temperature waves in the myocardium.

\section{The purpose and tasks of the study}

The purpose of the work is studying the conditions for the propagation of the temperature wave in the myocardium and development of a method for evaluating the rate of propagation of temperature waves in the process of controlled hypothermia and hyperthermia on the basis of non-invasive control of temperature on the surface of the heart.

To achieve this purpose, the following tasks were set:

1. To solve heat conduction equation taking into account the influence of heat sources in the myocardium, which cause the occurrence of temperature waves.

2. To determine a temperature function in the layer of the myocardium, as well as the amplitude and phase velocity of the temperature wave during cooling and warming of the heart.

3. To get a dependence of the rate of distribution of the heat flow in the myocardium from the temperature in the layer of myocardium in the process of hypothermia and hyperthermia.

\section{Materials and methods of research}

The method of evaluation the rate of propagation the temperature waves in the myocardium in the process of hypothermia and hyperthermia of the heart is realized on the basis of non-invasive control of temperature on the surface of the heart under artificial blood circulation. For realization the method of noncontact control of temperature and digital processing of video data thermograms in conditions of artificial blood circulation, the FLIR i7 thermocouple with a spectral range of 7,5-13 microns is used on the basis of non-cooling digital matrix elements $320 \times 240$ size, minimum focal length $0,6 \mathrm{~m}$ and temperature sensitivity $<1{ }^{\circ} \mathrm{C}$. The FLIR i7 thermograph allows to obtain a sequence of infrared heart images at a frequency of $9 \mathrm{~Hz}$, presented in the form of a sequence of frames. The obtained temperature distribution is the input data for solving the heat equation for temperature waves. The analysis of thermograms allows to determinate the function of distribution of temperature and rate of distribution of heat flux on the surface of the myocardium and at the depth of tissues in the process of hypothermia and hyperthermia of the heart under artificial blood circulation.

\section{Temperature waves in the theory of thermal conductivity}

For different degrees of temperature field heterogeneity, the flow velocity vector must satisfy the law of conservation of the mass of heat transferred, or the equation of continuity, which has the form [8]:

$$
\frac{\partial \rho}{\partial t}+\operatorname{div}(\rho \vec{u})=0
$$

where $\overrightarrow{\mathrm{u}}$ - vector of the heat flux rate, $\mathrm{m} / \mathrm{s}, \rho$ - the density of the myocardium, $\mathrm{kg} / \mathrm{m}^{3}, \mathrm{t}-$ the time of distribution of heat flow, s.

Thus, the generalized differential equation of heat conduction taking into account the convection flow in the myocardium takes the form [9]: 


$$
\left\{\begin{array}{l}
\rho C_{\mathrm{p}} \frac{\partial \mathrm{T}}{\partial \mathrm{t}}+\operatorname{div}\left(\rho \overrightarrow{\mathrm{u}} \frac{\partial \mathrm{T}}{\partial \mathrm{t}}\right)=-\operatorname{div}\left(\overrightarrow{\mathrm{q}}_{\mathrm{c}}\right)-\operatorname{div}\left(\overrightarrow{\mathrm{q}}_{\mathrm{R}}\right), \\
\frac{\partial \rho}{\partial \mathrm{t}}+\operatorname{div}(\rho \overrightarrow{\mathrm{u}})=0
\end{array}\right.
$$

where $\mathrm{C}_{\rho}$ - the specific heat of the myocardium, $\mathrm{J} /(\mathrm{kg} \times \mathrm{K}), \mathrm{q}_{\mathrm{C}}-$ the heat flux density due to heat transfer, $\mathrm{q}_{\mathrm{R}}$ - the density of the heat flow due to heat transfer, $\mathrm{W} / \mathrm{m}^{2}$.

In the general one-dimensional case, the properties of the myocardium and the parameters characterizing the heat flux can vary in the same direction in which the flow occurs - in parallel with the axis X (Fig. 1). In addition, the heat flux can change over time. Therefore, the density of the flow of heat $\mathrm{q}(\mathrm{x}, \mathrm{t})$ should be regarded as a function of coordinate $\mathrm{x}$ and time $\mathrm{t}$.

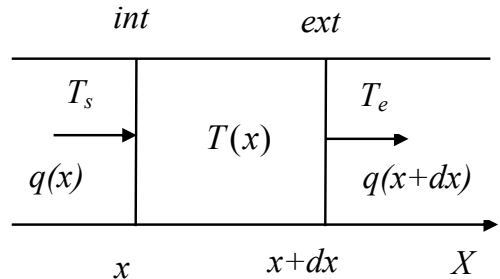

Fig. 1. The direction of the heat flow in the myocardium

To determine the thermal interaction at the boundary of two media, it is necessary to observe the condition equality of temperature and heat flows on both sides of the boundary of the section. The density of the heat flux $\mathrm{q}$ is a vector that coincides in the direction with the direction of heat propagation and is numerically equal to the amount of heat passing for one second through the area of the myocardium. To obtain the heat conduction equation for an infinitesimal area of the myocardium, one can consider a section of the surface in the form of a cylinder with forming, which is parallel to the axis $\mathrm{X}$ with a length $\mathrm{dx}$. The amount of heat $\mathrm{Q}_{\mathrm{int}}$ entering the cylinder due to the area of the cross section $\mathrm{S}$ in time dt, and the amount of heat $\mathrm{Q}_{\mathrm{ext}}$ coming out at the same time through the cylinder's base, is:

$$
Q_{\text {int }}-Q_{\text {ext }}=(q(x)-q(x+d x)) \cdot S \cdot d t=m_{\rho} d T,
$$

where $\mathrm{m}$ - mass of the cylinder (areas of the myocardium), $\mathrm{dT}$ - temperature change in myocardium.

If heat is not supplied through the lateral surface of the cylinder, then the total amount of heat entering the time $\mathrm{dt}$ through the examined part of the myocardium is determined by the formula:

$$
(q(x)-q(x+d x)) \cdot S \cdot d t=-\left(\frac{\partial q(x, t)}{\partial x}\right) \cdot S \cdot d x d t
$$

Equating both expressions and making reductions, we obtain the equation of heat conductivity for an infinitely small part of the myocardium in the one-dimensional case:

$$
\rho C_{\rho} \frac{\partial T}{\partial t}=-\frac{\partial q(x, t)}{\partial x}
$$

A heat flux occurs only when the temperature of the medium varies from one place to another. The simplest is the case of an infinite homogeneous medium - the thickness of the myocardium $\delta$. If on one border of the myocardium (int) temperature is maintained as $T_{s}$, but on the other (ext) temperature is $\mathrm{T}_{\mathrm{e}}$, and $\mathrm{T}_{\mathrm{s}}>\mathrm{T}_{\mathrm{e}}$, then the heat flux is proportional to the temperature $\Delta \mathrm{T}$. For infinite- 
ly thin layer and the axis $X$ is directed towards the lowering of temperature, then $\delta=d x, T=T(x)$, $\mathrm{T}_{\mathrm{e}}=\mathrm{T}(\mathrm{x}+\mathrm{dx})$, and the flow of heat in the myocardium is determined by the expression:

$$
\mathrm{q}_{\mathrm{s}}=-\gamma_{\rho} \frac{\left(\mathrm{T}_{\mathrm{e}}-\mathrm{T}_{\mathrm{s}}\right)}{\delta}=-\gamma_{\rho} \frac{\partial \mathrm{T}}{\partial \mathrm{x}},
$$

where $\Delta \mathrm{T}=\mathrm{T}_{\mathrm{s}}-\mathrm{T}_{\mathrm{e}}-$ temperature gradient in the myocardium in the direction of distribution of heat flow, ${ }^{\circ} \mathrm{C}, \gamma_{\rho}$ - coefficient of thermal conductivity of the myocardium, $\mathrm{W} /(\mathrm{m} \times \mathrm{K})$, which depends only on the physical properties of the myocardium.

The obtained formula is also valid in the case of an inhomogeneous medium with an arbitrary temperature distribution for all three spatial coordinates $\mathrm{x}, \mathrm{y}, \mathrm{z}$, if consider the infinitely thin layer of the myocardium perpendicular to the direction of the heat flow. Such layer of myocardium can be considered homogeneous, and its thermal conductivity $\gamma_{\rho}$ will be the function of all three spatial coordinates $\mathrm{x}, \mathrm{y}, \mathrm{z}$. In one-dimensional case, the thermal conductivity will depend only on one spatial coordinate $\mathrm{x}: \gamma_{\rho}=\gamma(\mathrm{x})$.

When the medium is homogeneous and the heat conductivity $\gamma_{\rho}$ does not depend on temperature, let's obtain the solution of the heat conduction problem for an infinite small and homogeneous portion of the myocardium from one spatial coordinate:

$$
\rho C_{\rho} \frac{\partial T}{\partial t}=-\frac{\partial^{2} T}{\partial x^{2}} \text { and } \frac{\partial T}{\partial t}=-\alpha \frac{\partial^{2} T}{\partial x^{2}}
$$

where $\alpha=\gamma_{\rho} / \rho C_{\rho}-$ temperature conductivity of the medium (myocardium).

If the sources of heat present in the myocardium, which is characteristic for the process of starting the heart, then the equation of heat conductivity for a homogeneous site has the form:

$$
\rho C_{\rho} \frac{\partial T}{\partial t}=-\frac{\partial^{2} T}{\partial x^{2}}+q_{j}(x)
$$

where $\mathrm{q}_{\mathrm{j}}$ - the amount of heat released by sources in the volume of the myocardium.

The heat can be released, for example, as a result of perfusion or passing of an electric current through a myocardium.

\section{The solution of the heat equation for temperature waves}

The temperature waves in the myocardium are periodic changes in the distribution of temperature in the environment, associated with periodic fluctuations in the density of heat flowing from the cooling and warming of the heart. If in a myocardium the temperature periodically changes in time, it leads to periodic changes of temperature in all other parts of the heart. The validity of this statement is due to the fact that the variables $\mathrm{x} i \mathrm{t}$ in the heat conduction equation, as well as the coefficient of thermal conductivity $\alpha$ are values of substance.

Let's accept the hypothesis that the temperature on the surface of the heart varies over time in a sinusoidal or cosine- law, fluctuating around some average value that is characteristic for convection of heat on the surface of the heart under artificial blood circulation. In the simplest case, when the myocardium is a homogeneous medium and fills the space limited by the plane $\mathrm{x}=0$, and the direction of heat transfer is the axis $\mathrm{X}$ directed inside the medium and perpendicular to its boundary, the average value of the temperature fluctuation around a certain set value can be assumed to be zero if agree to deduct from it the temperature.

Then the complex function of changing the temperature in the myocardium has the form:

$$
\mathrm{T}(\mathrm{x}, \mathrm{t})=\mathrm{T}_{\mathrm{s}}[\cos (\omega \mathrm{t}-\mathrm{kx})+\mathrm{i} \cdot \sin (\omega \mathrm{t}-\mathrm{kx})]=\mathrm{T}_{\mathrm{s}} \mathrm{e}^{\mathrm{i} \cdot(\omega \mathrm{t}-\mathrm{kx})},
$$

where $\mathrm{k}$ - constant value, $\omega$ - frequency change of direction of heat flow. 
The differentiation of the temperature change function $T(x, t)$ gives the following expressions:

$$
\frac{\partial \mathrm{T}}{\partial \mathrm{t}}=\mathrm{i} \omega \mathrm{T}_{0} \mathrm{e}^{\mathrm{i}(\omega \mathrm{t}-\mathrm{kx})}=\mathrm{i} \omega \mathrm{T}, \frac{\partial^{2} \mathrm{~T}}{\partial \mathrm{x}^{2}}=-\mathrm{k}^{2} \mathrm{~T}_{\mathrm{s}} \mathrm{e}^{\mathrm{i}(\omega t-\mathrm{kx})}=-\mathrm{k}^{2} \mathrm{~T}
$$

Substituting these expressions into the heat conduction equation, we obtain the dependence of the temperature conductivity of the medium (myocardium) on the frequency of temperature fluctuations $\omega$ when heat convection on the heart surface:

$$
\mathrm{i} \cdot \omega=-\alpha \cdot \mathrm{k}^{2} \text {. }
$$

Execution of this condition means that the complex function of temperature change $T(x, t)$ will be the solution of the heat equation for any value continuously $T_{s}$. If the value is $\omega$ is real and positive, then constant $\mathrm{k}$ will be a complex value, and may have two meanings:

$$
\mathrm{k}=\sqrt{-\mathrm{i} \omega / \alpha}= \pm \sqrt{\omega / 2 \alpha}(1-\mathrm{i}) .
$$

Since the temperature fluctuations begin to be excited on the surface of the myocardium and transmitted to the environment, then these oscillations should creep as far away from the surface of the heart. Therefore, physical meaning has only an expression for the function of change in the myocardium temperature of the following type:

$$
\mathrm{T}(\mathrm{x})=\mathrm{T}_{\mathrm{s}} \exp \left(-\sqrt{\frac{\omega}{2 \alpha}} \mathrm{x}\right) \operatorname{expi}\left(\omega \mathrm{t}-\sqrt{\frac{\omega}{2 \alpha}} \mathrm{x}\right) .
$$

From the complex solution for the function of change in the myocardium temperature it is possible to go to the substance form using the Euler formula:

$$
\mathrm{T}(\mathrm{x})=\left\{\begin{array}{l}
\mathrm{T}_{\mathrm{s}} \exp \left(-\sqrt{\frac{\omega}{2 \alpha}} \mathrm{x}\right) \cos \left(\omega \mathrm{t}-\sqrt{\frac{\omega}{2 \alpha}} \mathrm{x}\right), \\
\mathrm{T}_{\mathrm{s}} \exp \left(-\sqrt{\frac{\omega}{2 \alpha}} \mathrm{x}\right) \sin \left(\omega \mathrm{t}-\sqrt{\frac{\omega}{2 \alpha}} \mathrm{x}\right) .
\end{array}\right.
$$

If fix the coordinate $x$, then at each point of space the function of temperature $T(x, t)$ represents harmonic vibrations in time with the period $\tau=2 \pi / \omega$ and the phase of these oscillations, which varies from one part of the medium to another. Perturbations, which describe at each point of space a function of temperature change $T(x, t)$, represent a temperature wave propagating in a myocardium with a phase velocity $v$. Length of the temperature wave $\lambda$ is defined as the distance that passes through the wave for a period $\tau$ :

$$
\lambda=v \tau=2 \pi \sqrt{\pi \alpha \tau}
$$

The amplitude of the temperature wave decreases in the medium y e- times for each plot length $1=1 / \alpha=\lambda / 2 \pi$ in the direction of wave propagation:

$$
\mathrm{T}(\mathrm{x})=\mathrm{T}_{\mathrm{s}} \exp (-\Omega \cdot \mathrm{x}), \Omega=\sqrt{\omega / 2 \alpha}=2 \pi / \lambda,
$$

where $\Omega$ - coefficient of attenuation of the temperature wave.

Limit and initial conditions that satisfy the solution for the temperature change function $T(x, t)$, can be obtained if set the initial values $x=0$ and time $t=0$ : 


$$
\mathrm{T}_{\mathrm{t}=0}=\mathrm{T}_{\mathrm{s}} \exp \left(-\sqrt{\frac{\omega}{2 \alpha}} \mathrm{x}\right) \cos \sqrt{\frac{\omega}{2 \alpha}} \mathrm{x}, \quad \mathrm{T}_{\mathrm{x}=0}=\mathrm{T}_{\mathrm{s}} \cos \omega \mathrm{t}
$$

The heat equation retains the properties of linearity and homogeneity only in a given temperature range, in which the temperature conductivity $\gamma_{\rho}$ myocardium is a constant value.

\section{Temperature field in conditions of artificial blood circulation}

The distribution of the temperature field in the myocardium describes the temperature values for all points of space at a given time. Since in the real conditions of artificial blood circulation the initial distribution of temperature can be arbitrary, the problem setting for finding the boundary conditions may be as follows:

- on the myocardium surface at a time $\mathrm{t}=0$ harmonious fluctuations of temperature are excited, and then they are maintained indefinitely for a long time;

- no additional sources of heat other than blood coming from the contour of artificial blood circulation, inside the myocardium;

- at the end of a long period of time, all fluctuations of temperature in the environment are extinguished, except for forced oscillations;

- the forced oscillations will have the same periodicity in time as the temperature fluctuations on the surface of the myocardium.

The differential heat equation, which takes into account the rate of distribution of the heat flux in the medium for an infinitesimal area $v$ of the myocardium in the one-dimensional case, has the form:

$$
\rho \mathrm{C}_{\rho}\left(\frac{\partial \mathrm{T}}{\partial \mathrm{t}}+v \nabla \mathrm{T}(\mathrm{x}, \mathrm{t})\right)=-\nabla \mathrm{q}(\mathrm{x}, \mathrm{t})
$$

where $v$ - the rate of distribution of the heat flow in the myocardium.

If the heat flow occurs at one boundary of the medium (int) with surface temperature $T_{s}$, extends along the axis $\mathrm{X}$ and is supported to the other boundary of the environment (ext) with temperature $\mathrm{T}_{\mathrm{e}}<\mathrm{T}_{\mathrm{s}}$, then at any point of a homogeneous layer of myocardium it can be described by the following expression:

$$
-\gamma_{\rho} \frac{\partial \mathrm{T}}{\partial \mathrm{x}}=\mathrm{h}_{\mathrm{i}} \cdot\left(\mathrm{T}-\mathrm{T}_{\mathrm{e}}\right), \mathrm{h}_{\mathrm{i}}=\mathrm{C}_{\rho} \mathrm{dT}
$$

where $h_{i}$ - heat transfer coefficient for the $\mathrm{i}$ - point in the myocardium. Substituting this expression into the formula for the equation of heat conductivity, we obtain the solution of the problem in time in one spatial coordinate - the axis X.

Substituting this expression into the formula for the equation of heat conductivity, let's obtain the solution of the problem in time in one spatial coordinate - the axis X:

$$
-\frac{\partial}{\partial x}\left(\gamma_{\rho} \frac{\partial T}{\partial x}\right)_{x=i}=\frac{\partial}{\partial x}\left(-\gamma_{\rho} \frac{\partial T}{\partial x}+\rho h_{i} v_{i}\right)
$$

For a small part of the myocardium, which is an infinitely thin layer $\delta=\mathrm{dx}$ along the axis $\mathrm{X}$, the change in the temperature field will be expressed in terms of expression:

$$
\theta(x)=\frac{T-T_{s}}{T_{e}-T_{s}}=\left(\exp \left(\frac{\rho C_{\rho} v_{i}}{\gamma_{\rho}} x\right)-1\right) /\left(\exp \left(\frac{\rho C_{\rho} v_{i}}{\gamma_{\rho}} \delta\right)-1\right)
$$

where $v_{i}-$ the rate of distribution of heat flow for $i$ - points of the environment. 
Substituting this expression into the formula for the heat flow, let's obtain the heat transfer coefficient in the myocardium $h_{i}$ for the $i$ - point of the medium:

$$
\mathrm{h}_{\mathrm{i}}=\rho \mathrm{C}_{\rho} \mathrm{v}_{\mathrm{i}} /\left(\exp \left(\frac{\rho \mathrm{C}_{\rho} \mathrm{v}_{\mathrm{i}}}{\gamma_{\rho}} \delta\right)-1\right)
$$

Provided if in the selected temperature interval $\mathrm{dT}=\mathrm{T}_{\mathrm{s}}-\mathrm{T}_{\mathrm{e}}$ given temperature of myocardium $\gamma_{\rho}$ and the coefficient of heat transfer in the myocardium $h_{i}$ is a constant, the differential equation of heat conductivity has the properties of linearity and homogeneity. This allows to solve the heat conduction equation with respect to the rate of distribution of the heat flow in the myocardium:

$$
v(x, T)=-\frac{h}{\rho C_{\rho}} \frac{T_{e}-T_{s}}{T-T_{s}} W\left(-\exp \left(-\frac{h}{\gamma_{\rho}} \frac{T_{e}-T_{s}}{T-T_{s}} x\right)+1\right)
$$

where $W(x, t)$ is Lambert's function.

If limit the values $x \geq-1 / e$ and believe that $W(x, t) \geq-1$, then Lambert's function is defined as an unambiguous function.

The solution to the problem of thermal conductivity shows that in the surface layer of the myocardium, temperature fluctuations can be established with a period $T=2 \pi \omega$, which corresponds to the period of thermal waves of heat sources. Temperature fluctuations in the surface layer of the myocardium occur with a phase shift [10], so the depth of penetration of heat into the surface of the myocardium depends on the period of temperature fluctuations on the heart surface. The amplitude of the temperature wave in the surface layer is proportional to the depth:

$$
\mathrm{T}(\mathrm{x})=\mathrm{T}_{\mathrm{s}} \cdot \exp (-\omega \sqrt{\alpha} \cdot \chi),
$$

where $\chi$ is time delay of maxima and minima of the temperature in the myocardium.

Taking into account the process of penetration of temperature waves into the depth of the surface layer of the myocardium let's obtain an expression for changing the temperature field, which depends on the frequency of temperature variations $\omega$ :

$$
\frac{\mathrm{T}(\mathrm{x})-\mathrm{T}}{\mathrm{T}}=\exp \left(-\sqrt{\frac{\omega}{2 \alpha}} \mathrm{x}\right)-1 \text {. }
$$

The ratio shows that the longer the period of temperature fluctuations, the less depth of penetration of temperature waves in the depth of the myocardium [11]. A solution for the heat conduction problem taking into account the temperature waves in one spatial coordinate relative to the rate of distribution of the heat flow in the myocardium $v$ will be described by expression of the form:

$$
\begin{aligned}
& v(x, \omega, T)=-\frac{h}{\rho C_{\rho} \theta(x)} \frac{T_{s}}{T-T_{s}}\left(\exp \left(-\sqrt{\frac{\omega}{2 \alpha}} x\right)-1\right) \times \\
& \times W\left(-\exp \left(-\frac{h}{\gamma_{\rho}} \frac{T_{e}-T_{s}}{T-T_{s}} x\right)+1\right) .
\end{aligned}
$$

Provided that the differential equation of heat conductivity has the properties of linearity and homogeneity, the expression for the rate of propagation of the heat flux has the form:

$$
\begin{aligned}
& v(x, \omega, T)=-\frac{h}{\rho C_{\rho}} \frac{T_{s} \Delta T}{\left(T-T_{s}\right)^{2}}\left(\exp \left(-\sqrt{\frac{\omega}{2 \alpha}} x\right)-1\right) \times \\
& \times W\left(-\exp \left(-\frac{h}{\gamma_{\rho}} \frac{T_{e}-T_{s}}{T-T_{s}} x\right)+1\right) .
\end{aligned}
$$




\section{Results of studies on the distribution of temperature in the myocardium}

During controlled cooling and warming, the uneven distribution of temperature in tissues is manifested. In inflammatory processes in the myocardium, temperature zones are defined, in which the temperature difference with the surrounding tissues is recorded. With a chronic inflammation, the temperature difference is $0,7-1{ }^{\circ} \mathrm{C}$, with acute illness $1-1,5{ }^{\circ} \mathrm{C}$, and when the destructive process reaches $1,5-2{ }^{\circ} \mathrm{C}[12,13]$. The propagation of the temperature wave on the myocardium surface can be observed in the process of hypothermia (Fig. 2) and hyperthermia (Fig. 3) of the heart, consistently recorded in conditions of artificial blood circulation [14].

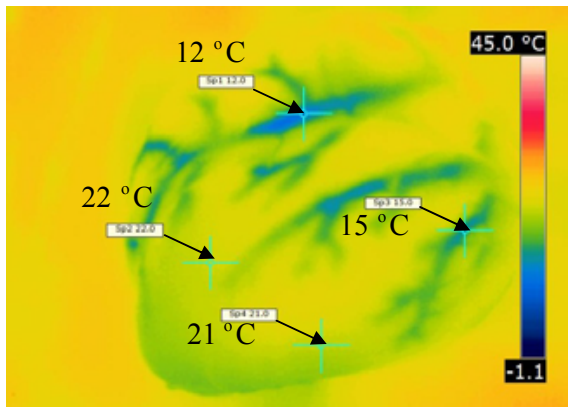

Fig. 2. Thermographic representation of temperature wave propagation on the surface of the myocardium for isolated heart in the process of controlled hypothermia

In the process of hypothermia and hyperthermia of the heart using a thermal imager, the temperature on the surface of the myocardium was not invasively recorded [15]. The temperature data on the surface of the myocardium (Fig. 2, 3) show the presence of a temperature difference in the coronary channel $2-3{ }^{\circ} \mathrm{C}$, which indicates the heterogeneity of the temperature distribution on the myocardium surface under artificial blood circulation.

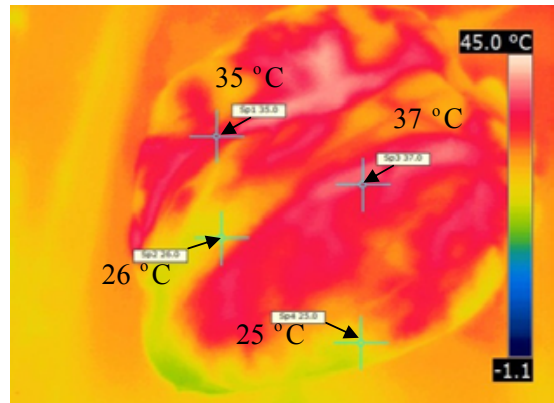

Fig. 3. Thermographic representation of the temperature wave propagation on the myocardium surface for the isolated heart in the process of controlled hyperthermia

As for the distribution of the temperature field, the initial temperature of the myocardium, recorded at the beginning of the process of cooling the isolated heart, was not less $21-22^{\circ} \mathrm{C}$ and no more $25-26^{\circ} \mathrm{C}$ at the beginning of warming up the heart. The temperature gradient between the tissues of the myocardium and the coronary solution is from $6^{\circ} \mathrm{C}$ and more (to $10{ }^{\circ} \mathrm{C}$ ). When warming the heart, the coronary bed in the myocardium stands out at a temperature gradient from $9{ }^{\circ} \mathrm{C}$ and more (to $12{ }^{\circ} \mathrm{C}$ ).

The evaluation of the rate of propagation of temperature waves in the myocardium can be made on the basis of the obtained equation for the rate of the heat flux distribution $v(x, T)$ in moments of time $t$, which correspond to the completion of the processes of hypothermia and hyperthermia. For calculation of the rate of distribution of heat flow in the myocardium $v(x, T)$ the values of constant coefficients and physical parameters of tissues [16] and the values of the temperature of the walls of the myocardium were used:

$\mathrm{T}_{\mathrm{s}}=23^{\circ} \mathrm{C}$ - temperature of the external wall of the myocardium,

$\mathrm{T}_{\mathrm{e}}=17^{\circ} \mathrm{C}$ - temperature of the internal wall of the myocardium. 
The rate of flow of heat in the myocardium depending on the temperature on the surface of the myocardium and the cooling depth along the axis X shown in Fig. 4.

The obtained dependences (Fig. 4) for the rate of the heat flow distribution in the myocardium $v(x, T)$ show that the magnitude of the rate of distribution of the heat flow in the absence of additional sources of heat in the myocardium is practically unchanged with the depth of penetration of the heat flow in the myocardium. The distribution of heat flux significantly depends substantially depends on the temperature on the surface of the heart, it increases in exponential law from the value $v=0,1 \times 10^{-3} \mathrm{~m} / \mathrm{s}$ to size $v=1,5 \times 10^{-3} \mathrm{~m} / \mathrm{s}$ with temperature rise.

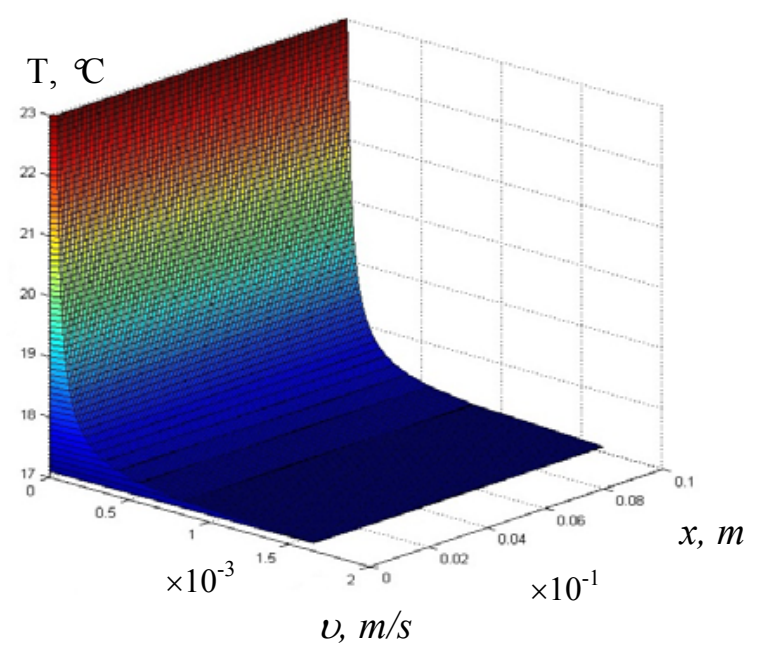

Fig. 4. Dependence of the rate of the heat distribution flow $v(x, T)$ in the myocardium from the temperature $T$ in the depths $x$ layer of myocardium.

The source of heat sources in the myocardium may be temperature waves due to uneven flow of cooled or warmed blood due to rotation of the rollers of the pump of the artificial blood circulation device. Accelerated the rate of distribution of heat flow in the depth of the myocardium $v(\mathrm{x}, \omega, \mathrm{T})$ taking into account temperature waves $\mathrm{T}(\mathrm{x})$ in the surface layer on one spatial coordinate, depending on the temperature difference between the outer and inner heart of the heart, is shown in Fig. 5.

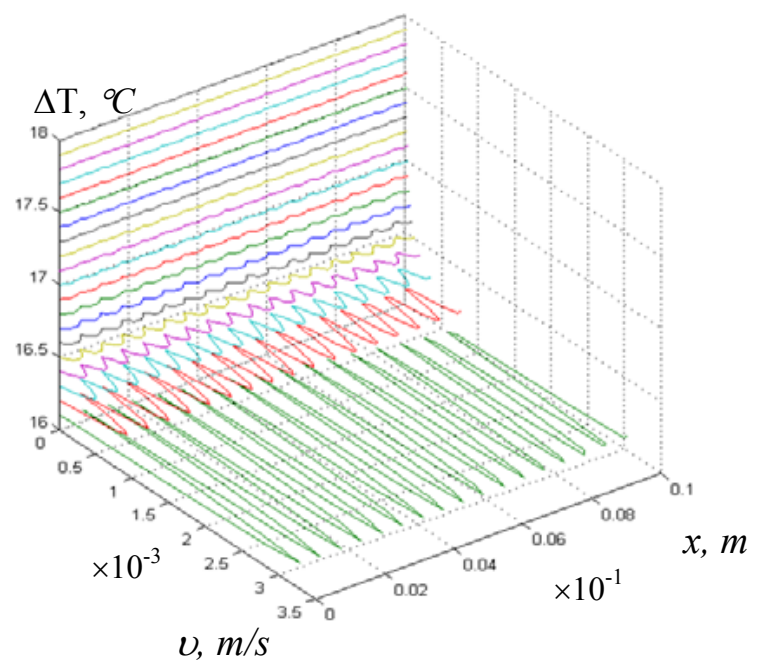

Fig. 5. Dependence of the rate from distribution of heat flow $v(x, \Delta T)$ in the depths of $x$ layer in temperature difference $\Delta \mathrm{T}$ between the outer and inner walls of the myocardium, taking into account the temperature waves 
Obtained dependencies (Fig. 5) for the rate of distribution of heat flow in the myocardium $v(x, \omega, T)$ show that for the frequency $\omega=250 \mathrm{rpm}$ the amplitude of the temperature waves is maximal in the surface of the myocardium and significantly decreases in the direction of the propagation of the heat flux. Moreover, the rate of distribution of the heat flow in the myocardium significantly decreases from the value $v=2,5 \times 10^{-3} \mathrm{~m} / \mathrm{s}$ at gradient $\Delta \mathrm{T}=16^{\circ} \mathrm{C}$ to size $\mathrm{v}=0,1 \times 10^{-3} \mathrm{~m} / \mathrm{s}$ at gradient $\Delta \mathrm{T}=16,5^{\circ} \mathrm{C}$.

If to take into account that during the operation of the apparatus of artificial blood circulation the blood flow may warm up with a temperature gradient $0,2-0,5^{\circ} \mathrm{C}$ or cool with a large gradient $0,5-1,7^{\circ} \mathrm{C}$ then, taking into account the temperature waves in the homogeneous layer of the myocardium, the temperature between the external one $\mathrm{T}_{\mathrm{e}}$ and inside $\mathrm{T}_{\mathrm{s}}$ the wall of the myocardium changes the faster, the higher the density of the heat flow $\mathrm{q}_{\mathrm{s}}$ through the surface with the thickness $\delta$.

\section{Conclusions}

The overall reactivity and course of pathological processes in the myocardium are largely determined by the rate of propagation of temperature waves in the tissue layer. Moreover, the temperature difference between the blood in the contour and the patient's body should not differ by more than $15^{\circ} \mathrm{C}$. The temperature gradient between the blood in the vessels and the myocardium leads to a temperature difference between the outer and inner layer of the heart (Fig. 4), which, according to the function of the rate of propagation of the heat flux, changes in exponential law.

Periodic changes in the temperature in the operating field and in the circulatory circuit lead to the appearance of temperature waves on the surface and in the depth of the surface layer of the myocardium.

The estimated values of the rate of propagation of temperature waves in the myocardium under artificial blood circulation correspond to the density of the flow of heat in the process of hypothermia and hyperthermia of the heart, the regulation of which ensures the survival of the myocardium during the operation. Further studies of temperature wave propagation in the heart under artificial blood circulation should be directed to increasing the effectiveness of non-invasive methods for controlling temperature in order to determine the extent and location of contours of the coronary arteries, which will allow reliable and effective determination of the degree of absolute and relative violations of myocardial blood supply.

\section{References}

[1] Gurev, V., Lee, T., Constantino, J., Arevalo, H., Trayanova, N. A. (2010). Models of cardiac electromechanics based on individual hearts imaging data: Image-based electromechanical models of the heart. Biomechanics and Modeling in Mechanobiology, 10 (3), 295-306. doi: 10.1007/s10237010-0235-5

[2] Vetter, F. J., McCulloch, A. D. (1998). Three-dimensional analysis of regional cardiac function: a model of rabbit ventricular anatomy. Progress in Biophysics and Molecular Biology, 69 (2-3), 157-183. doi: 10.1016/s0079-6107(98)00006-6

[3] Nickerson, D., Smith, N., Hunter, P. (2005). New developments in a strongly coupled cardiac electromechanical model. Europace, 7, 118-127. doi: 10.1016/j.eupc.2005.04.009

[4] Helm, P. A., Tseng, H.-J., Younes, L., McVeigh, E. R., Winslow, R. L. (2005). Ex vivo 3D diffusion tensor imaging and quantification of cardiac laminar structure. Magnetic Resonance in Medicine, 54 (4), 850-859. doi: 10.1002/mrm.20622

[5] Lunkenheimer, P. P., Redmann, K., Kling, N., Jiang, X., Rothaus, K., Cryer, C. W. et. al. (2006). Three-dimensional architecture of the left ventricular myocardium. The Anatomical Record Part A: Discoveries in Molecular, Cellular, and Evolutionary Biology, 288 (6), 565-578. doi: 10.1002/ar.a.20326

[6] Vadakkumpadan, F., Arevalo, H., Prassl, A. J., Chen, J., Kickinger, F., Kohl, P. et. al. (2010). Image-based models of cardiac structure in health and disease. Wiley Interdisciplinary Reviews: Systems Biology and Medicine, 2 (4), 489-506. doi: 10.1002/wsbm.76 
[7] Helm, P., Beg, M. F., Miller, M. I., Winslow, R. L. (2005). Measuring and Mapping Cardiac Fiber and Laminar Architecture Using Diffusion Tensor MR Imaging. Annals of the New York Academy of Sciences, 1047 (1), 296-307. doi: 10.1196/annals.1341.026

[8] Sivukhin, D. V. (2005). Obshhiy kurs fiziki. Vol. 2. Termodinamika i molekulyarnaya fizika. Moscow: Fizmatlit/MFTI, 544.

[9] Lobasova, M. S., Finnikov, K. A., Milovidova, T. A., Dekterev, A. A., Serebrennikov, D. S., Minakov, A. V. (2009). Teplomassoobmen. Krasnoyarsk: IPK SFU, 295. Available at: http://files.lib.sfu-kras. ru/ebibl/umkd/1536/u_lecture.pdf

[10] Irodov, I. E. (1999). Volnovye protsessy. Osnovnye zakony. Moscow: Laboratoriya bazovykh znaniy, 256

[11] Pushkareva, A. E. (2008). Metody matematicheskogo modelirovaniya v optike biotkani. Saint Petersburg: SPbGU ITMO, 103.

[12] Kotovskyi, V. Y. (2009). Obgruntuvannia vymoh do umov provedennia termohrafichnykh doslidzhen biolohichnykh ob yektiv. Visti akademii inzhenernykh nauk Ukrainy, 2 (39), 6-11.

[13] Nicholas, A., Diakides, B., Joseph, D., Bronzino, A. (2008). Medical Infrared imaging. London: CRC Press Taylor Group LLC, 451.

[14] Ghosh, S., Falter, F., Cook, D. J. (Eds.) (2009). Cardiopulmonary Bypass. New York: Cambridge University Press, 207. Available at: https://suny-perfusion-knowledge.wikispaces.com/file/view/Cardiopulmonary+bypass+book+-+Ghosh.pdf

[15] Shlykov, V., Danilova, V., Maksymenko, V., Maryna, S. (2017). Application of Model of Heat Exchange for Miocardium Provided Stationary Convection Laminar Flow. Journal of Cardiology \& Current Research, 10 (1). doi: 10.15406/jccr.2017.010.00350

[16] Lokshin, L. S., Lur'e, G. O., Dement'eva, I. I. (1998). Iskusstvennoe i vspomogatel'noe krovoobrashhenie v serdechno-sosudistoy khirurgii. Moscow: Nauchnyy tsentr khirurgii, Rossiyskaya akademiya meditsinskikh nauk, 93. 